\title{
1 A mechanism to prevent transformation of the Whi3 mnemon into a prion.
}

2 Authors: Yasmin Lau ${ }^{1}$, luliia Parfenova ${ }^{2}$, Juha Saarikangas ${ }^{3,4,5}$, Richard A. Nichols ${ }^{1}$, Yves

3 Barral $^{2 *}$ and Fabrice Caudron ${ }^{1^{*}}$

4

5 Affiliations:

$6 \quad 1$-School of Biological Sciences, Queen Mary University of London, London, UK.

72 - Institute of Biochemistry, ETH Zürich, Zürich, Switzerland.

83 - Helsinki Institute of Life Science HiLIFE, Helsinki, Finland.

94 - Faculty of Biological and Environmental Sciences, Helsinki, Finland.

105 - Neuroscience Center, University of Helsinki, Helsinki, Finland.

\section{*Correspondence to: f.caudron@qmul.ac.uk or yves.barral@bc.biol.ethz.ch}

\section{Abstract:}

In response to deceptive courtship, budding yeast cells escape pheromone induced cell cycle arrest through coalescence of the G1/S inhibitor Whi3 into a dominant inactive superassembly. Strikingly, Whi3 super-assemblies remain stable over many cell cycles in the mother cells and are not passed on to the daughter cells. Thereby, Whi3 coalescence encodes memory, conferring to it the property of a mnemon (Whi $3^{\mathrm{mnem}}$ ), a protein which conformational change maintain a trait that is permanent in the mother cell but is not inherited by daughter cells. Mnemons share structural features with prions, which are self-templating protein conformations that are inherited by daughter cells. Yet, how the maintenance and asymmetric inheritance of Whi $3^{m n e m}$ are achieved is unknown. Here, we report that Whi3 ${ }^{m n e m}$ is closely associated with endoplasmic reticulum (ER) membranes and retained in the mother cell by the presence of lateral membrane diffusion barriers at the bud neck. Strikingly, barrier defects made $W_{h i} 3^{m n e m}$ propagate in a mitotically stable manner, like a prion. Alike Whi3 ${ }^{\text {mnem }}$, transformation of Whi3 into a prion required its poly-glutamine prion-like domain. Thus, we propose that Whi $3^{m n e m}$ is in a self-templating state, lending temporal stability to the memory that it encodes, while its anchorage into the compartmentalized membranes of the ER ensures its confinement in the mother cell and prevents its infectious propagation. These results term encoding of information. 


\section{Introduction}

Prions are proteins that can adopt several conformations with at least one of them that is selftemplating, lending it a self-perpetuating character. Prion conversion is essential for physiological processes including innate immunity (Hou et al., 2011) and the generation of phenotypic diversity of single celled organisms ranging from bacteria to yeast (Coustou et al., 1997; True and Lindquist, 2000; Yuan and Hochschild, 2017). Beyond physiology, prions were initially discovered as agents of devastating diseases in humans (Kuru, Creutzfeldt-Jakob's disease) and animals (Scrapie and Bovine spongiform encephalopathy) (Prusiner, 1982). Moreover, prion-like behavior of different proteins may be linked to other diseases including Alzheimer's and Parkinson's diseases (Goedert, 2015), as well as resistance to anti-fungal drugs (Suzuki et al., 2012). What mechanisms control prions and particularly their selftemplating activity is still an open question.

Prions are common protein elements and several have been identified in the budding yeast S. cerevisiae (Wickner et al., 2015) allowing a wealth of research to understand their regulation and function (Harvey et al., 2018). The protein Sup35 can adopt a conformation to form the $[P S /+]$ prion, the best characterized to date (Tuite and Cox, 2006). Sup35 is a translation terminator that loses its function in its $[P S /+]$ form, resulting in stop codon readthrough and phenotypic diversity (True and Lindquist, 2000). Importantly, Sup35 contains a prion-like domain, defined as rich in Glutamine and Asparagine ( $Q / N)$ residues and depleted of charged and hydrophobic residues (Alberti et al., 2009). This domain drives the Sup35 prion state by adopting a self-templating conformation (Serio et al., 2000) and mediating the assembly of Sup35 into amyloid fibrils. Once established, fibrils formed by the Sup35 protein are fragmented into smaller seeds by the protein disaggregase Hsp104 (Narayanan et al., 2006). The self-templating activity of prion seeds converts most of the protein pool to the prion form and the seeds are free to diffuse to the daughter cells. Therefore, prions behave as infectious particles, establishing $[\mathrm{PS} /+]$ not only in the cell in which it originated but also in all its daughter cells and hence the growing colony. In silico analysis of the yeast proteome has identified around 200 proteins in S. cerevisiae containing a potential prion-like domain (Alberti et al., 2009). However, we still do not know if all prion-like domains can adopt self-templating conformations and whether all these proteins function as prions.

Whi3 contains two prion-like domains, composed of PolyQ- and PolyN-rich streches (Alberti et al., 2009; Caudron and Barral, 2013). Whi3 is an mRNA binding protein involved in the timing of the G1/S transition of the cell cycle and the control of cell size in $S$. cerevisiae (Garì et al., 2001). The function of Whi3 that has been studied the most is the regulation of the G1/S transition of the cell cycle through binding and inhibiting the translation of the mRNA that 
encodes the $\mathrm{G} 1$ cyclin CLN3. This inhibition ensures that entry into $\mathrm{S}$ phase is delayed until cells reach a critical size. The prion-like domains of Whi3 are required for Whi3 to adopt a conformation that releases Whi3's inhibition on CLN3 mRNA and allows a long-lasting phenotypic switch. Upon exposure to mating pheromone, haploid yeast cells arrest in the G1 phase of the cell cycle and grow towards the source of pheromone. This cytoplasmic projection is termed a shmoo. After prolonged exposure of pheromone without a mating partner in reach, yeast cells become refractory to the pheromone signal and resume their cell cycle (Caudron and Barral, 2013; Moore, 1984). They switch from a shmooing phase to a budding phase. Remarkably, once established, this pheromone refractory state is stable, lending memory to the cell that there is no partner available. As a consequence, these cells keep on budding for the remainder of their life span even in the presence of mating pheromone. Strikingly, daughter cells do not inherit this adaptation and restore their ability to shmoo in response to pheromone upon separation from their mother cell (Caudron and Barral, 2013). Whi3's prion-like domains trigger both escape from pheromone arrest and the memory state by mediating the condensation of Whi3 into super-assemblies.

Whi3s' domain organization and ability to switch between soluble and condensed phases are features common to prions such as Sup35. The pheromone refractory state is very stable once it has been established, even when pheromone is removed from the medium. The deletion of either prion-like domain of Whi3 results in a late escape from pheromone arrest that is not stably maintained in the mother cells. In other words, cells can switch back and forth to a shmooing and a budding phase in the presence of pheromone. Moreover, when cell extracts are run on a semi-denaturing gel, Whi3 appears to be mostly monomeric in untreated cells and mostly found in large, homo-multimeric assemblies in cell treated with pheromone (Caudron and Barral, 2013). These data point to a possible self-templating activity of Whi3 in cells that are exposed to pheromone.

Importantly, despite many shared features with known prions, the mode of inheritance of Whi3 super-assemblies is different from the inheritance of prions during cell division (Caudron and Barral, 2013; Tuite, 2016). Indeed, the Whi3 super-assemblies remain in the mother cells at mitosis, ensuring that the memory state is stable in the mother cell and is not propagated to the progeny. On the contrary, prions infect the bud of a dividing yeast cell. Given its role in cellular memory and its behavior being so distinct from prions, Whi3 was termed a mnemon $\left(\right.$ Whi3 $\left.{ }^{\text {mnem }}\right)$.

While the prion-like domains of Whi3 ${ }^{\text {mnem }}$ are important for escape from pheromone arrest, we do not know the mechanism of memory stability and if this involves a self-templating activity of Whi3 ${ }^{m n e m}$. If this is the case, what makes mnemons distinct from prions is unclear. In this 
103 work, we wondered whether the confinement of Whi3 ${ }^{m n e m}$ to the mother cell requires the 104 presence of lateral membrane diffusion barriers. Diffusion barriers are membrane specialized 105 domains that limit the diffusion of membrane associated structures across cellular 106 appendages (Caudron and Barral, 2009; Saarikangas and Barral, 2011) including the primary 107 cilium (Hu et al., 2010; Kim et al., 2010), dendritic spines (Ewers et al., 2014) and the sperm 108 tail (Toure et al., 2011). In budding yeast, diffusion barriers form at the bud neck in the ER 109 membranes and the nuclear envelope during closed mitosis. Their formation depends on 110 septins, a family of cytoskeletal protein forming filaments (Mostowy and Cossart, 2012) at the 111 bud neck (Luedeke et al., 2005), proteins involved in sphingolipid biosynthesis including the 112 sphinganine C4-hydroxylase Sur2 (Clay et al., 2014) and proteins involved in polarized cell 113 growth such as the actin nucleation promoting factor Bud6 (Graziano et al., 2013, 2011) and 114 the small GTPase Bud1/Rsr1 (Bender and Pringle, 1989). Diffusion barriers in yeast limit the 115 diffusion of ageing factors, such as nuclear pores, misfolded proteins, age-induced protein 116 deposits and DNA circles from the mother cell to the bud (Clay et al., 2014; Denoth-Lippuner 117 et al., 2014; Saarikangas et al., 2017; Shcheprova et al., 2008).

118 In light of these data, we first asked whether membrane diffusion barriers contribute to $119 W^{2} 3^{m n e m}$ asymmetric behaviour during cell division. 


\section{Results}

133 Cells lacking diffusion barriers can acquire a novel pheromone refractory state.

134 Growth of yeast colonies was monitored on solid medium containing low concentration of 135 pheromone (10nM). Wild type cells grew slowly while cells that hardly escape pheromone 136 arrest (whi3- $\Delta p Q$, (Caudron and Barral, 2013)) grew very poorly (Figure 1A). Both strains grew 137 equally well on a solid medium that does not contain pheromone. On medium containing 138 pheromone, wild type cells initially shmoo and resume cell division after several hours. Their 139 daughter cells behave similarly, first shmooing and then resuming cell division. This slows 140 down the production of daughter cells and explains why wild type cells grow slowly in the presence of pheromone. In contrast, whi3- $\Delta p Q$ mutant cells keep on shmooing for a very long

142 time before resuming cell division and many cells will not even escape pheromone induced 143 cell cycle arrest. Therefore, whi3- $\Delta p Q$ mutant cells grow even slower on pheromone 144 containing plates. To test for a role of diffusion barriers in the compartmentalization of the 145 pheromone refractory state, we monitored the growth of several mutant strains with impaired 146 lateral diffusion barriers. We hypothesized that if diffusion barriers are involved in the 147 confinement of Whi3 $3^{m n e m}$ these mutant strains may grow differently on pheromone. Some of 148 the bud1 $\Delta$, bud $6 \Delta$ and sur2 $\Delta$ mutants grew as wild type and other much better on pheromone containing plates, even at high pheromone concentration $(0.6 \mu \mathrm{M}$, Figure $1 \mathrm{~A})$. At such a high concentration of pheromone, cells cannot escape pheromone arrest, suggesting that colonies growing in such conditions had acquired a strong resistance to pheromone. Colonies growing on high pheromone concentration kept their resistance to pheromone after several rounds of streaking; we termed these isolates as constitutive escapers (CE). To measure how frequent CE are, we plated at least 44 independent clones for each mutation obtained from heterozygous diploid on rich media that do not contain pheromone or contain a high pheromone concentration $(0.6 \mu \mathrm{M})($ Figure $1 \mathrm{~A}-\mathrm{B})$. Frequency of CE appearance was highly variable between individual clones of wild type strains (between $1.11 \times 10^{-7}$ and $5.3 \times 10^{-2}$ ) with a median of $1.36 \times 10^{-5}$ cells. Phenotypic mutation rate for sterile mutants was measured in a bar1 $\Delta$ background at $3.07 \times 10^{-6} /$ genome/generation (Lang and Murray, 2008). We found that it was also variable in sur2 $\Delta$ clones but the median was significantly increased 3.13 times compared to wild type (4.26 $\times 10^{-5}$, Figure $\left.1 \mathrm{~B}\right)$. In bud $6 \Delta$ and bud $1 \Delta$ mutant cells, frequencies again varied extensively between clones and the median frequency was significantly increased 6.07 times $\left(8.27 \times 10^{-5}\right)$ and 3.51 times $\left(4.78 \times 10^{-5}\right)$ respectively, compared to wild type. We analyzed these results differently in an attempt to standardize the variances. The ratios were logit transformed (see material and methods for more details). Having fitted the average for each yeast strain, the residuals were clearly strongly asymmetrical (Supplemental Figure 1). This pattern might be expected if in a subset of cases the constitutive escaper 
phenotype occurred early in the culture. Since the incidence of these outcomes did not differ between strains, they were excluded from subsequent analysis of the ratios. Figure $1 \mathrm{C}$ shows the distribution of the logit transformed ratios for each genotype. A linear model describing the means of each strain, showed a highly significant difference between wild type and diffusion barrier mutants (ANOVA $\mathrm{p}<2 \mathrm{e}-16$ ). These data suggest that the frequency increase of constitutive escapers is related to the disruption of the diffusion barrier. Moreover, the fact that we measured very variable frequencies and many of them being well above mutation rate suggest that CE are induced by an epigenetic mechanism. Since escape from pheromone arrest involves Whi3 ${ }^{\mathrm{mnem}}$, we considered the possibility of a prion being the molecular basis of the CE phenotype.

Upon microscopic observation of CE, we noticed that some CE isolates seemed to have a small cell size. Therefore, we measured the cell size of several CE. While wild type cells and sur2 $\Delta$ parental strains had comparable cell size (5.6 $\mu \mathrm{m}$, Figure 1D), sur2 ${ }^{C E}$ displayed isolates which were on the large range or on the small range of cell sizes. Remarkably, the small cell sized sur2 ${ }^{C E}$ were comparable in size to cells deleted for WHI3 either in a wild type or in a sur2 $\Delta$ background (Figure 1D). This prompted us to ask whether Whi3 could be a prion in CE isolates and if it was required for the formation of CE. Classically, prion forms of proteins lose the function of the native conformation. Thus, if Whi3 was losing its function in a prion form, we would expect that deleting WHI3 would result in a CE phenotype. The frequency of CE in whi3 $\Delta$ mutant strains was increased compared to wild type $\left(2.45\right.$ times, $\left.3.33 \times 10^{-5}\right)$ and further increased in whi3 $\Delta$ sur2 $\Delta$ strains (4.58 times, $6.23 \times 10^{-5}$ ). This result indicate that Whi3 inactivation is not sufficient to make cells refractory to pheromone, although it may contribute to it. We next wondered whether Whi3 was required to maintain the CE phenotype. We supposed that if Whi3 was acting as a dominant negative form in these cells, deleting $W H I 3$ in sur2 ${ }^{C E}$ would restore pheromone arrest. We specifically deleted $W H I 3$ in several sur $2 \Delta^{C E}$ that had a small cell size. This deletion did not restore pheromone arrest (not shown) suggesting that maintenance of the CE phenotype is not dependent on Whi3 or that Whi3 super-assemblies are not the only dominant factor repressing pheromone response in CEs. Altogether, we observe that whi3 $\Delta$ cells are not all CE (most of the cells arrest upon exposure to pheromone) yet deleting WHI3 seems to favor CE formation. However, Whi3 is not required to maintain the CE phenotype. One interpretation of these results may be that WHI3 inactivation is one step for cells to become CE and that somehow, the diffusion barrier inhibits the transition to the CE state.

To further test whether Whi3 may be in a prion form in CE, we isolated 20 CE from a sur2 $\Delta$ parental strain (sur2 $\Delta^{C E}$ ) expressing Whi3-GFP from its endogenous locus on plates containing high pheromone concentration and we observed the localization of Whi3-GFP in 
these cells. In parallel, we obtained cell size measurements of these isolates. In the parental sur2 $\Delta$ strain, Whi3-GFP localized rather diffusely throughout the cytoplasm and to a few granules ((Caudron and Barral, 2013; Garì et al., 2001) and Figure 1E). In small cell sized sur2 $\Delta^{C E}$, Whi3 localized diffusely throughout the cytoplasm and to brighter foci that were substantially bigger and more intense than the granules shown by the parental strain. These bright foci were much less frequent in large cell sized CE (Figure 1E). Remarkably, these bright foci localized both to the mother and bud compartments of dividing cells. This is in contrast to Whi $3^{\mathrm{mnem}}$ super-assemblies which are formed in the mother cell and do not typically appear in the buds (Caudron and Barral, 2013). Therefore, in small cell sized sur2 $\Delta^{C E}$, Whi3 tends to adopt a localization pattern reminiscent to that of the classical yeast prion Sup35, in its [PS/+] form (Derdowski et al., 2010). We contemplated the possibility that if Whi3 needs to be in a prion form for cells to become $\mathrm{CE}$, it would require its prion-like domain. To test this hypothesis, we estimated the frequency of $C E$ in whi3- $\Delta p Q$ mutant clones. CE frequency was remarkably lower in these cells than in any other strain we tested $\left(6.25 \times 10^{-6}, 2.18\right.$ times smaller than wild type strains, Figure $1 \mathrm{~B}$, and significantly lower than wild type using the logit transformed data, $p<0.0008$, Figure $1 C$ ). Deleting $B U D 1$ in a whi3- $\triangle p Q$ strain did not increase the frequency of CE much $\left(7.45 \times 10^{-6}, 1.192\right.$ times higher than in $B U D 1$ whi3- $\Delta p Q$ mutant strain) and was lower than in bud1s mutant cells.

Altogether, we found that disruption of the diffusion barrier increases the appearance of CE, that many of them are small cell sized and display a localisation of Whi3 to bright foci. The formation of CE is increased when WHI3 is deleted but decreased in strains that lack the prionlike domain of Whi3. This suggest that Whi3 is indeed in a prion form and that inactivation of Whi3 is a major contribution to the formation of CE. However, since the frequency of CE can be still very high in CE that lack the prion-like domain of Whi3, it is very likely that other factors are involved, possibly novel, yet undiscovered, prions.

To test whether the molecular events leading to the formation of CE is indeed caused by prions, we tested how growth on high pheromone concentration segregated at meiosis. Prions segregate in a non-Mendelian manner at meiosis and are usually passed on to all of the four meiotic products. Consistent with their extensive resistance to pheromone treatment, crossing the CE to wild type cells was inefficient. However, we could backcross some of them and dissect the obtained tetrads. Meiosis gives rise to 4 spores, 2 of which are MAT $\alpha$ and 2 MATa. Therefore, we expected that out of 4 spores, 2 would always grow on alpha-factor because they are MAT $\alpha$. In wild type cells, the 2 other spores do not grow on alpha-factor $(0.6 \mu \mathrm{M}$, Supplemental Figure 2). If in the CE more than 2 spores were growing, it would mean that they had inherited the CE phenotype. If the CE is due a mutation that is unlinked to the MAT 
240 locus, 4/6 of the tetrads should have 3 spores growing on alpha-factor containing medium, 1/6

241 should contain 4 such spores and the last 1/6 of the tetrads should contain only 2 of them

242 (pattern \#1). If it is due to a mutation linked to the MAT locus, the fraction of tetrads with 3 and

2434 spores growing on alpha-factor containing medium should be increased (pattern \#2). In case

244 of a non-mendelian factor propagating through meiosis, all tetrads should contain 4 spores

245 growing on alpha-factor containing medium (pattern \#3). Finally, a non-mendelian factor that

246 does not pass meiosis should produce tetrads with always only 2 spores growing on alpha-

247 factor containing medium (pattern \#4). We backcrossed 13 independent CE strains and tested

248 the growth of each spore on alpha-factor $(0.6 \mu \mathrm{M})$. For 4 backcrosses, we observed tetrads in

249 which 2, 3 or 4 spores out of 4 were growing on $\alpha$-factor (Supplemental Figure 2). These

250 backcrosses fall in the pattern \#1, suggesting the presence of a single 'sterile' mutation

251 segregating independently from the mating type locus. In 4 other backcrosses, the majority of

252 the tetrads contained 2 spores and few 3 spores growing on alpha-factor containing medium,

253 most compatible with the pattern \#4. Furthermore, 5 backcrosses followed strictly pattern \#4

254 (Supplemental Figure 2). Thus, the last 9 backcrosses, which are not compatible with a single

255 mutation, suggests that the CE phenotype is due to non-mendelian factor that is lost during

256 meiosis. To further test if these CE traits are based on a prion-like mechanism, we tested

257 whether they were cured upon inhibiting different prion effectors. We isolated 31 sur2 $\Delta^{C E}$ and

258 passaged them three times on YPD, YPD supplemented with guanidine hydrochloride ( $3 \mathrm{mM}$ )

259 to inhibit Hsp104 (Ferreira et al., 2001; Tuite et al., 1981) or YPD supplemented with radicicol

$260(10 \mu \mathrm{M})$ to inhibit Hsp90 (Sharma et al., 1998). In addition, we transformed all 31 sur2 $\triangle^{\mathrm{CE}}$ and

261 the parental sur2 $\triangle$ strain with a dominant negative allele of SSA1 (SSA ${ }^{D N}$, (Brown and

262 Lindquist, 2009; Jarosz et al., 2014)). In all cases, after 3 passages the 31 sur2 $\Delta^{C E}$ were still

263 able to grow on YPD containing pheromone $(0.6 \mu \mathrm{M})$, while the parental sur2 $\Delta$ strain was not

264 (Supplemental Figure 3). However, upon microscopic observation of sur2 $\Delta^{C E 1}$, we found that 265 many cells were shmooing and other dividing. This was not the case for other sur $2 \Delta^{C E}$, and it 266 was also not the case for sur2 $\Delta^{C E 1}$ passaged on YPD without drugs or with $\mathrm{GuHCl}$ or radicicol 267 (Supplemental Figure 3). Therefore, the CE phenotype is not cured by either $\mathrm{GuHCl}$, radicicol 268 or passages for many generations on YPD, but one variant was partially cured upon inhibition 269 of the Hsp70 chaperone Ssa1.

271 Altogether, our data suggest that the majority of the CE formed in cells lacking a diffusion 272 barrier at the bud neck are due to a non-mendelian factor and that for about at least half of 273 them Whi3 is contributing to their formation by behaving not anymore as a mnemon but as a prion. 
277 Because diffusion barriers seem to play a role in the transformation of Whi3 into a prion, we next asked whether diffusion barriers also play a role in prion induction and curing. We specifically focused on the best studied prion in yeast, $[P S /+]$. We previously observed that farnesylation of the Hsp40 co-chaperone Ydj1 was required to lower premature stop codon read-through in the sequence coding for GFP in $[P S /+]$ cells (Saarikangas et al., 2017). Strength of codon read-through was linked to the potential collection of prion seeds to ER membranes. We therefore analyzed stop codon readthrough in sur2 $\Delta$ cells. Using flow cytometry, the fluorescence intensity of strains that express a GFP allele containing a premature stop codon was measured (as in Saarikangas et al., 2017). Deletion of SUR2 did not change the fluorescence intensity of these cells (Figure 2A). We next probed for a role of lateral diffusion barriers in the de novo appearance and curing of $[P S /+]$ prions. There was no significant difference $(p=0.5204$, t-test $)$ in the $[P S /+]$ appearance frequencies in wild type and sur2 $\Delta$ strains (Figure $2 \mathrm{~B}$ ). We also analyzed the dynamics of $[P S /+]$ curing by Guanidine Hydrochloride ( $\mathrm{GuHCl}, 3 \mathrm{mM}$ ) in wild type and sur2 $\Delta$ cells and observed very similar dynamics of curing (Figure 2C). Moreover, stop codon readthrough was comparably lower in wild type and sur2 $\Delta$ cells treated with $0.1 \mathrm{mM} \mathrm{GuHCl}$ and $1 \mathrm{mM} \mathrm{GuHCl}$ demonstrating that we could quantitatively measure the strength of stop codon readthrough in these experiments (Figure $2 A)$. Altogether, these data establish that compartmentalization of endo-membranes by a diffusion barrier at the bud neck does not control the inheritance of Sup35 prion seeds during cell division.

Whi3 super-assemblies and Sup35 foci differ in their association to ER membranes.

Because diffusion barriers affect Whi3 transformation into a prion and not [PS/+], we wondered if Sup35 and Whi3 associated to different extends with ER membranes. We first analyzed how Sup35-GFP, in its $[P S /+]$ prion form, localized relative to the ER visualized using the translocon subunit Sec61 tagged with mCherry as a marker. Sup35-GFP formed several small foci in dividing cells and we found that most of these foci were spatially excluded from the Sec61 signal (Figure $3 A$ and $B, 53.1 \pm 10.7 \%$ of Sup35-GFP foci were away from the Sec61mCherry signal). We also noticed that $100 \%$ of the cells contained at least one focus away from ER membranes (Figure $3 \mathrm{C}$ ). This may explain why curing and induction of $[P S /+]$ is not affected in sur2 $\Delta$ mutant cells. Next, we analyzed the localization of Whi3-mNeonGreen (Whi3-mNG) in its mnemon form relative to Sec61-mCherry. Contrarily to Sup35-GFP, in cells that were exposed to pheromone for 3 hours Whi3-mNG super-assemblies were mostly apposed to ER membranes ( $72.5 \pm 4.1 \%$ of super-assemblies, Figure $3 \mathrm{D}$ and $\mathrm{E})$. Moreover, 
311 the fraction apposed to the ER system increased upon longer times of pheromone exposure $312(82.7 \pm 2.1 \%$ after 4 hours, $90.0 \pm 3.5 \%$ after 6 hours and $92.1 \pm 3.2 \%$ after 10.5 hours, Figure $3133 \mathrm{D}$ and E). Remarkably, while $41.2 \pm 9.1 \%$ of the cells had at least one Whi3-3GFP super314 assembly away from the ER after 3 hours of pheromone treatment, this value dropped to 27.7 $315 \pm 10.7 \%, 20.0 \pm 1.7 \%$ and $14.8 \pm 4.9 \%$ after 4 hours, 6 hours and 10.5 hours of pheromone 316 treatment respectively (Figure 3F). Finally, we analyzed the localization of Whi3-mNG relative 317 to ER membranes in small bud6 $\Delta^{C E}$. We found that $60.27 \%$ of the Whi3-mNG foci were close 318 to ER membranes in bud6 $\Delta^{C E}$ cells, in stark contrast to the granules in non-CE bud6 6 cells 319 which were much more strongly associated to ER membranes (88.17\%, Figure $3 \mathrm{G}$ and $\mathrm{H})$.

320 Altogether, we conclude that association of Whi3 ${ }^{\mathrm{mnem}}$ with ER membranes is tighter with time 321 after escape form pheromone arrest and that a major difference between Whi $3^{\text {mnem }}$ superassemblies and the prion form of Sup35 or the Whi3 foci in CE is their different link to the ER membranes network.

Endoplasmic reticulum compartmentalization is required for the retention of Whi3 ${ }^{m n e m}$ and the pheromone refractory state in the mother cell

The establishment and the maintenance of the pheromone refractory state is facilitated by Whi3's conformational change and super-assembly formation. We wondered whether mother cells with defective diffusion barriers were more likely to pass Whi3 super-assemblies to their daughters. Therefore, we treated cells expressing WHI3 fused to 3 superfolding Green Fluorescent Proteins in tandem (Whi3-3GFP) with pheromone for 5 hours and then released them in a pheromone free medium for 1.5 hours to allow mother cells to produce a bud. The mother and bud pairs were imaged and the localization of Whi3-3GFP analyzed. Most wild type mother cells contained a super-assembly of Whi3 ${ }^{\text {mnem, }}$ and we observed similar results in bud $6 \Delta$, sur2 $\Delta$ and bud1 $1 \Delta$ mutant cells. However, while only $24.00 \pm 6.22 \%$ of the wild type buds had a super-assembly, $62.24 \pm 3.85 \%$ of the bud $6 \Delta, 64.01 \pm 3.65 \%$ of the sur $2 \Delta$ and 65.26 $\pm 4.15 \%$ of the bud1 $1 \Delta$ mutant buds already had at least one (Figure $4 \mathrm{~A}-\mathrm{B}$ ). These data are consistent with a role for diffusion barriers in the retention of Whi3 ${ }^{\mathrm{mnem}}$ super-assemblies in the mother cells that escaped pheromone arrest.

340 We then tested whether diffusion barriers are required for the retention of the pheromone refractory state in the mother cell. We exposed haploid MATa cells to pheromone as before

342 (Caudron and Barral, 2013). Wild type cells initially all shmooed upon $7 \mathrm{nM} \alpha$-factor treatment 343 and $90.5 \%$ of the cells escaped pheromone arrest with an average timing of $7.35 \pm 3.00$ hours 344 (Figure 5A-B). Mother cells kept this refractory state faithfully, $100 \%$ of the initial mother cells 
did not produce a second shmoo after escaping pheromone arrest. We previously found that roughly $90 \%$ of the daughter cells from mothers that have escaped from pheromone arrest undergo shmooing in response to pheromone, upon separation from their mother in the presence of pheromone (Caudron and Barral, 2013). We refined our analysis here by separating data depending on whether daughters were the first or subsequent daughters after escape from pheromone arrest. We observed that nearly $50 \%$ of the first daughter cells fail to respond to pheromone after birth, while this number drops to $14.2 \%, 13.3 \%, 6.9 \%$ and $4.4 \%$ for the subsequent $2^{\text {nd }}, 3^{\text {rd }}, 4^{\text {th }}$ and $5^{\text {th }}$ daughters of the same mother cell (Figure $5 \mathrm{C}$ ). In order to assess whether the retention of the pheromone refractory state in the mother cell is indeed dependent on the presence of diffusion barriers at the bud neck, we tested sur2 2 , bud6 $6 \Delta$ and bud $1 \Delta$ mutant cells. For reasons that remain unknown at this stage, cells with a sur $2 \Delta$ mutation escaped from pheromone arrest slightly earlier than wild type cells (5.9 \pm 2.0 hours, Figure 5 A-B). Once these mother cells escaped, they maintained the pheromone refractory state as efficiently as wild type cells and did not shmoo again. However, the fraction of sur2 $\Delta$ daughter cells that failed to shmoo after separating from their mother cell was increased 1.51, 2.83 and 1.66 folds for the $1^{\text {st }}, 2^{\text {nd }}$ and $3^{\text {rd }}$ daughters respectively. (Figure $5 \mathrm{C}$ ). The bud6 $\Delta$ mutant cells responded to pheromone as well as wild type cells and escaped pheromone arrest slightly later than them (Figure 5A-B). As for the sur2 $\Delta$ daughter cells, many bud6 $6 \Delta$ daughter cells failed to shmoo upon separation from their mother cell (Figure $5 \mathrm{C}$ ). We obtained similar results with bud1 $1 \Delta$ mutant cells (Figure 5A-C). These results indicate that the ER diffusion barrier reinforces the confinement of the pheromone refractory state to the mother cell.

Next, we wondered whether bud6s, sur $2 \Delta$ or bud1 $1 \Delta$ mutations had any effect on the timing of escape in the daughter cells that do shmoo in response to pheromone. We reasoned that these daughter cells may escape faster if they inherit factors promoting escape from their mother cell. However, we did not detect any general effect on the timing of daughter cells escape in these mutants (Figure 5D). We still observed that sur2 $\Delta$ daughter cells escaped faster than wild type, bud6 6 and bud1 $\Delta$ cells, as their mothers do.

Altogether, we conclude that diffusion barrier in the cortical ER helps confining the pheromone refractory state to the mother cell. Importantly, in cells lacking a functional barrier the inheritance of the refractory state by the daughter cells does not take place at the cost of the mother cell losing the pheromone refractory state. Indeed, bud $6 \Delta$, sur2 $\Delta$ and bud $1 \Delta$ mutant mother cells maintained this state as efficiently as wild type cells. Together these results indicate that the ER diffusion barrier confines the pheromone refractory state to the mother cell by preventing the formation of super-assemblies in daughter cells. Strikingly, this role of the barrier is most evident in the first few cycles after pheromone escape by the mother cell and much less so later on. 
382 The prion-like domain of Whi3 is required for symmetric inheritance of the refractory state in

383 cells with an impaired diffusion barrier.

384 We next reasoned that if inheritance of the pheromone refractory state by daughter cells 385 depends on Whi3 ${ }^{\text {mnem }}$ super-assemblies, then preventing the conversion of Whi3 to its 386 mnemon form may lead to less daughter cells inheriting the pheromone refractory state upon 387 diffusion barrier defects. To test this idea, we exposed whi3- $\Delta p Q$, whi3- $\Delta p Q$ bud6 $\Delta$ and whi3$388 \Delta p Q$ bud1 $\triangle$ mutant cells to pheromone and analyzed whether the daughters of cells escaping 389 pheromone arrest were shmooing or budding after birth. Most whi3- $\Delta p Q$ daughter cells

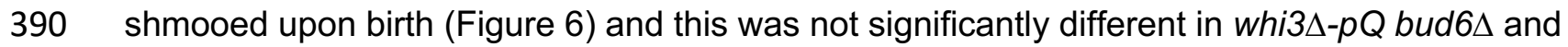
391 whi3 $\Delta-p Q$ bud1 $\Delta$ cells. Taken together, these data argue that the Q-rich prion-like domain of 392 Whi3 is involved in the inheritance of the pheromone refractory state by the daughters of cells 393 that lack a diffusion barrier. 


\section{Discussion}

410 In this work, we investigated the mechanisms that allow the pheromone refractory state to be 411 stable in the mother cells over many divisions while preventing it to being passed on to 412 daughter cells. Our data support a model in which Whi3 adopts a self-templating conformation 413 that depends on its prion-like domains upon prolonged pheromone exposure. The self414 templating Whi $3^{m n e m}$ is confined by diffusion barriers to the mother cell and when this 415 confinement is lost, Whi3 ${ }^{\text {mnem }}$ does propagate to daughter cells. In a substantial fraction of the 416 cases, it even starts to propagate in a mitotically stable manner, similarly to prions.

417 Indeed, we found that yeast cells can acquire a mitotically stable refractory state to pheromone 418 and cells carrying this state were termed constitutive escapers. Some of these CE are small 419 cell sized, phenocopying a loss of function of Whi3. In these small cell sized CE Whi3 forms 420 foci, a hallmark of prion localization in yeast. Moreover, CE induction requires the prion-like 421 domains of Whi3. These data indicate that Whi3 is in a dominant inactive, self-templating prion 422 form in these CE variants. The fact that deleting WHI3 increases CE frequency indicates that 423 an important step of the transition to the CE state is the inactivation of Whi3 through its

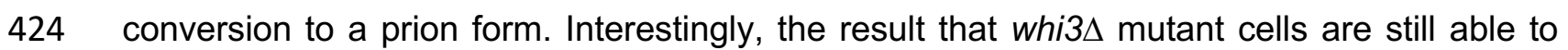
acquire the CE state indicates the existence of additional prion-like factors in CE cells. Therefore, CE is a promising case for identifying novel prions in yeast.

427 During escape from pheromone arrest in diffusion barrier deficient cells, inheritance of the 428 pheromone refractory state by daughter cells requires the prion like domains of Whi3, also 429 indicating that Whi3 $3^{\mathrm{mnem}}$ is in a self-templating conformation. In diffusion barrier deficient cells, 430 the pheromone refractory state is stable in the mother cells, suggesting that small assemblies are diffusing to daughter cells and seeding the pheromone refractory state there. However, in diffusion barrier defective cells only the first two to three daughter cells can inherit the pheromone refractory state efficiently. This is much less frequent in the next daughter cells. We propose that within the time of two to three cell cycles the super-assemblies mature to a more solid form that no longer generate seeds or captures them efficiently. These data provide experimental support for a mechanism of memory maintenance through a self-templating mechanism. This is a particularly interesting mechanism because it can establish a stable memory that lasts longer than the turnover of the Whi3 protein itself and prevents newly synthesized Whi3 to restore WHI3 function.

All these data argue that Whi3 is able to form several types of assemblies, either being in a mnemon or in a prion form. Since, CE can have different cell sizes, it could also be that Whi3 can adopt several types of prion forms, which is reminiscent of the different strains prions can form (Derkatch et al., 1996). It may also be that the mnemon and the prion forms are actually 
444 the same and that what is lost are the mechanisms of confinement to the mother cell. CE were

445 frequent in cells with defective diffusion barriers. Because bud1 $1 \Delta$ cells display this feature, our

446 results point to the ER diffusion barrier playing an important role in the prevention of CE

447 induction. Indeed, only the ER diffusion barrier is affected by BUD1 deletion, while the nuclear

448 diffusion barrier remains intact (Clay et al., 2014). Yet, these observations beg the question of

449 how diffusion barriers could prevent the induction of CE?

450 We found that $[P S /+]$ prion induction and curing is not affected by whether the diffusion barriers 451 are present or not. On the contrary, we observed that Whi $3^{\text {mnem }}$ super-assemblies and the 452 pheromone refractory state they encode are not confined as efficiently to the mother cell in 453 diffusion barrier defective cells than in wild-type cells. Thus, we propose that one function of 454 the diffusion barriers is to retain Whi3 ${ }^{\mathrm{mnem}}$ super-assemblies in the compartment where they are formed. Even though Whi3 is not known to be lipidated or having transmembrane domains, Whi3 associates with ER membranes (Vergés et al., 2007). Regardless of how it does so, such an association would allow the diffusion barrier to limit the diffusion of Whi3 seeds along ER membranes through the bud neck. More importantly, we found that Whi3 ${ }^{\text {mnem }}$ superassemblies are closely colocalizing with ER membranes during prolonged pheromone response. It will be therefore important in the future to understand how Whi3 is anchored at ER membranes to be able to test our model of retention by the ER diffusion barrier more thoroughly. Altogether a feature of mnemons may be their tight association with ER membranes allowing confinement to one cellular compartment. This is strikingly different to the Sup35 prion, most of which is detected in foci that are away from ER membranes. In this case, the presence of diffusion barriers in the ER membrane has no impact on exchange of seeds across the bud neck. Once Sup35 acquires its prion form in a cell, it can freely invade the bud and maintain the phenotypes associated with it in the progeny, and hence, the whole colony formed. We observed a similar localization pattern respective with ER membranes for Whi3 foci in CE cells. This suggests to us that the Whi3 prion form may differ from the mnemon form at least in part through their membrane association status. Whether the detachment of

471 Whi3 in CE from ER membranes stems from a conformational change inhibiting Whi3 472 interaction with its ER anchor or from a change in the regulation of this interaction will need to 473 be clarified.

474 Altogether, we propose that the absence of the barrier allows for the selection of stable prion 475 strains of Whi3 and possibly other prions. The emergence of these prions would not be able 476 to emerge if they are confined.

477 Many proteins can adopt prion-like behavior across all kingdom of life. Recent effort to 478 characterize these proteins have suggested that this is probably more widespread than 
479 anticipated. We presented here that yeast cells have evolved mechanisms to confine and thus

480 control some of them. We propose that this is not restricted to budding yeast. A case could be

481 made for the protein CPEB, which adopt a prion-like conformation at activated dendritic spines

482 (Khan et al., 2015; Si et al., 2010). Interestingly, dendritic spines are highly compartmentalized

483 cellular appendages, and similarly to dividing yeast cells, their compartmentalization and

484 morphology is controlled by septin proteins at the spine neck (Ewers et al., 2014). It may

485 therefore be that CPEB prion conversion is confined to an activated dendritic spine through

486 its compartmentalization by diffusion barriers, which could prevent the spreading of the prion

487 form to the neighbouring non-activated spines. Another consideration may also be that

488 confinement of prion-like elements such as mnemons may prevent their transformation into

489 infectious prion particles. In this case, we could envision that pathologies involving prion-like

490 behaviors may arise when confinement of these elements is lost.

491

492

493

494

495

496

497

498

499

500

501

502

503

504

505 


\section{Strains}

509 Strains used for escape from pheromone arrest were derivatives of the s288c BY4743 wild

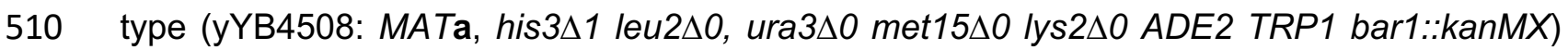

511 with deletions obtained according to (Janke et al., 2004) (yYB4507: sur2::NAT; yYB4510:

512 bud6::NAT; yYB4511: bud1::NAT). Strains used for Whi3 localization were derivatives of the 513 wild type (yYB6520: MATa ura3 $\triangle 0$, his $\Delta 13$, leu2 $\Delta 0$, TRP1, LYS2, ADE2, met15 0 , WHI3514 3GFP:kanMX, bar1::HIS3) backcrossed in yYB4510 for bud6 $\Delta$ (yYB10190), in yYB4507 for 515 sur2 $\Delta$ (yYB10192) or in yYB4511 for bud1 $\Delta$ (yYB10187) strains. Strains for the co-localization 516 of Whi3-3GFP and Sec61-mCherry were obtained by PCR tagging of Sec61 in yYB6520 517 (yFC203: MATa ura3 $\Delta 0$, his $\Delta 13$, leu2 $\Delta 0$, TRP1, LYS2, ADE2, met15 0 , WHI3-3GFP:kanMX, 518 bar1::HIS3, Sec61-mCherry:kanMX). W303 strains used to test for [PSI+] induction and curing 519 were obtained from Jonathan Weissman, yYB8040 (MAT $\alpha$, leu2-3,-112; his3-11,-15; trp1-1; 520 ura3-1; ade1-14; can1-100, [RNQ+], [PS/+]). The sur2 $\Delta$ strain was obtained by deleting SUR2 521 according to Janke et al. (Janke et al., 2004) (yYB8435 MAT $\alpha$, leu2-3,-112; his3-11,-15; trp1522 1; ura3-1; ade1-14; can1-100, sur2::HIS3, [RNQ+], $[P S /+])$. Localisation of Whi3 in sur2 ${ }^{C E}$ 523 was analysed in strain yYB5147 (his3 $\Delta 1$, leu2 $\Delta 0$, met15 $\Delta 0$, ura3 $\Delta 0, L Y S 2$, bar1::kanMX, 524 sur2::NatMX, Whi3-GFP:HIS3MX6). To analyse co-localisation of Sup35-GFP and Sec61525 mCherry we used strain yFC202 (MATa/alpha SUP35/SUP35-GFP:HIS3; leu2-3,-

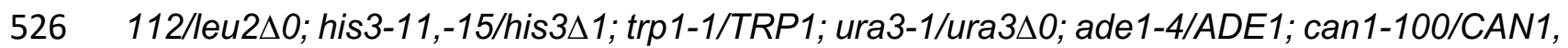
527 MET15/met15 20 , SEC61/Sec61:mCherry:URA3 [RNQ+], [PS/+]). For stop codon 528 readthrough, we used wild type (MATalpha, leu2-3-112, his3-11,-15, trp1-1, ura3-1, ade1-4, 529 can1-100, [RNQ+], [PSI+], pRS304 pGPD GST-UGA-GFP-pest:URA3) or sur2A strain 530 (MATalpha, leu2-3-112, his3-11,-15, trp1-1, ura3-1, ade1-4, can1-100, [RNQ+], [PSI+], 531 pRS304 pGPD GST-UGA-GFP-pest:URA3 sur2::KanMX).

532

534 All images were acquired either on a Personal Deltavision (Applied Precision) equipped with 535 a CCD HQ2 camera (Roper) and 250W Xenon lamps controlled by Softworx or a Deltavision 536 Elite (GE Healthcare) equipped with a sCMOS camera and solid-state light-emitting diodes 537 controlled by Softworx. Fluorescein isothiocyanate and tetramethylrhodamine isothiocyanate 538 filters were used for imaging GFP and mCherry fluorescence. Deconvolution was performed 539 using Softworx. 
541 Experiments were carried out with the ONIX microfluidic perfusion platform with Y04C

542 microfluidic plates (CellAsic). Medium was yeast extract peptone dextrose (YPD)

543 supplemented with $20 \mathrm{mg} / \mathrm{ml}$ casein and containing $7 \mathrm{nM} \alpha$-factor (Figure 2 and 3E) or $6 \mathrm{nM}$

$544 \alpha$-factor (Figure 3C-D).

\section{Quantification of Shmooing}

546 Shmooing or budding states were inspected visually. During microfluidic experiments, images 547 were taken every $15 \mathrm{~min}$. Unbudded cells that showed a polarized growth were counted as 548 shmooing. Unbudded cells undergoing isotropic growth were counted as G1 cells. Usually 549 these cells soon started forming a bud. Samples consisted of three independent clones.

551 Cells were grown in YPD supplemented with $20 \mathrm{mg} / \mathrm{ml}$ casein and containing $7 \mathrm{nM} \alpha$-factor 552 and were briefly centrifuged at $600 \mathrm{~g}$, resuspended in SD-TRP medium, placed between slide 553 and coverslip, and imaged immediately. Images were analyzed after deconvolution with 554 Softworx software as before [27]. Three clones with total $n>122$ cells were observed for each 555 strain.

\section{Quantification of [PSI+] de novo appearance}

$557[\mathrm{PSI}+][\mathrm{PIN}+]$ wild type and sur2 $\Delta$ cells were first cured with 3 passages on YPD agar medium 558 containing $3 \mathrm{mM} \mathrm{GuHCl}$. Red single colonies [psi-] [pin-] were assessed for their ability to 559 become white again. Cells were plated on SC-Ade and YPD and the frequencies of 560 appearance of white colonies were measured. White colonies were tested for their ability to 561 become red again after passages on YPD containing $3 \mathrm{mM} \mathrm{GuHCl}$.

\section{Quantification of [PSI+] curing during treatment with GuHCI}

563 Cells were grown overnight in liquid YPD and diluted in the morning to $\mathrm{OD}_{600 \mathrm{~nm}}=0.2$ in YPD 564 with $3 \mathrm{mM} \mathrm{GuHCl}$. Samples were taken every 30 minutes and plated on synthetic medium with low adenine concentration. Liquid cultures were kept in exponential phase during the experiment. Colonies were allowed to grow at $30^{\circ} \mathrm{C}$ for several days and the proportion of white and red colonies was assessed after 2 days of incubation at $4^{\circ} \mathrm{C}$ to allow for the red colour to develop well. We initially determined that curing started to happen after 12 hours of

$569 \mathrm{GuHCl}$ treatment for both wild type and sur2 $\Delta$ strains. 


\section{Quantification of Stop-codon read-through by flow cytometry}

572 For the stop codon read through experiments, wild-type $[P S /+]$ and sur2 $\Delta[P S /+]$ cells 573 expressing chromosomally integrated pGPD GST-UGA-GFP-pest were grown for 5 hours 574 without $\mathrm{GuHCl}$ or with $0.1 \mathrm{mM}$ or $1 \mathrm{mM} \mathrm{GuHCl}$. The GFP fluorescence intensity was measured 575 with a BD Accuri C6 Flow Cytometer using 488 nm laser and 533/30 BD filter for 100000 576 cells/ clone (3 clones each). The data was analyzed using FlowJo software (FlowJo LLC).

\section{Quantification of CE frequencies}

578 Diploid strains heterozygous for the different mutations were sporulated (for example 579 SUR2/sur2 $\Delta$ or WHI3/whi3A). MATa spores were selected and their genotypes determined by 580 growth on selection media. Strains were grown in YPD until mid-log phase and spotted on 581 solid YPD and solid YPD containing $0.6 \mu \mathrm{M} \alpha$-factor. Colonies were counted after 2-3 days of 582 growth at $30^{\circ} \mathrm{C}$.

583 The analysis was conducted on the estimates of the of relative yeast density obtained from 584 the colony count $(C)$ at an appropriate dilution (D). The relative density for each clone was obtained in the presence of the pheromone (p) and the corresponding control (c), so the relative performance is given by the ratio:

$$
\mathrm{R}=\mathrm{CpDp} / \mathrm{CcDc}
$$

The ratio was logit transformed in an attempt to standardize the variance. Having fitted the average for each yeast strain, the residuals were clearly strongly asymmetrical (Supplemental

590 Figure 1 shows the deviation from a cumulative normal distribution). This pattern might be expected if in a subset of cases the constitutive escaper phenotype occurred early in the culture. The departure from a cumulative normal distribution of residuals is abrupt for standardized residuals greater than one (shown by the vertical line). Since the incidence of these outcomes did not differ between strains $\left(\chi^{2}=3.02, P=0.88\right)$ they were excluded from subsequent analysis of the ratios.

596 Figure 1C shows the distribution of the logit transformed ratios $(R)$ for each genotype. A linear 597 model describing the means of each strain, showed a highly significant difference between 598 the three strains for which there were a priori expectations of a stronger effect of the 599 pheromone (whi3- $\Delta p Q$, bud1 $\Delta$ whi3- $\Delta p Q$ and the wildtype) and the remainder (ANOVA P < $6002 \mathrm{2}-16)$. The fitted values for these two categories is shown by the red line. There were no 601 significant differences between the means for this remaining group $(P=0.41)$ whereas there 602 were significant differences among the three $(P<0.0007)$ - in particular whi3- $\Delta p Q$ was 
603

604

605

606

607

608

609

610

611

612

613

614

615

616

617

618

619

620

621

622

623

624

625

626

627

628

629

630

631

632

markedly lower than the wild type $(P<0.0008)$. The $R$ package for this analysis is available in the supplementary material.

\section{Cell sizes measurements}

Cell sizes were determined using a CASY cell counter model TTC (Schärfe system). Strains were grown to early log phase, diluted in CASYton (Electrolyte buffer from Schärfe system) and processed according to the manufacturer instructions.

\section{Quantification of Sup35 foci and Whi3 super-assemblies/foci/granules co-localisation with ER membranes}

For Whi3-3GFP localisation, cells were grown in YPD supplemented with $20 \mathrm{mg} / \mathrm{ml}$ casein and containing $7 \mathrm{nM} \alpha$-factor and were briefly centrifuged at $600 \mathrm{~g}$, resuspended in SD-TRP medium, placed on a SC-TRP agar pad covered by a coverslip, and imaged immediately. Images were analysed after deconvolution with Softworx software. Three clones with total $\mathrm{n}=$ 194 cells, 250 cells, 170 cells and 153 cells for 3 hours, 4 hours, 6 hours and 10.5 hours condition were observed for each strain. A total of 333, 402, 393 and 317 super-assemblies were analysed at 3 hours, 4 hours, 6 hours and 10.5 hours' time points. Note that we only analysed super-assemblies that were in the 5 best focal planes as co-localisation was difficult to assess on the top and bottom focal planes and we only counted Sup35-GFP foci and Whi33GFP super-assemblies in the mother cells.

\section{Acknowledgements}

We would like to thank Robin Hannay for his help in creating some of the strains. This work was supported by a Biotechnology and Biological Sciences Research Council Project grant (BB/S001204/1) to FC, QMUL to FC and YL, the Academy of Finland (317038) and Sigrid Jusélius Foundation to JS and the European Research Council (BarrAge 250278) and ETH Zurich (to J.S., F.C. and Y.B.). 


\section{References}

634

635

636

637

638

639

640

641

642

643

644

645

646

647

648

649

650

651

652

653

654

655

656

657

658

659

660

661

662

663

664

665

666

667

668

669

670

671

672

673

674

675

676

Alberti S, Halfmann R, King O, Kapila A, Lindquist S. 2009. A systematic survey identifies prions and illuminates sequence features of prionogenic proteins. Cell 137:146-158. doi:10.1016/j.cell.2009.02.044

Bender A, Pringle JR. 1989. Multicopy suppression of the cdc24 budding defect in yeast by CDC42 and three newly identified genes including the ras-related gene RSR1. Proc Natl Acad Sci U S A 86:9976-9980.

Brown JCS, Lindquist S. 2009. A heritable switch in carbon source utilization driven by an unusual yeast prion. Genes Dev 23:2320-2332. doi:10.1101/gad.1839109

Caudron F, Barral Y. 2013. A super-assembly of Whi3 encodes memory of deceptive encounters by single cells during yeast courtship. Cell 155:1244-1257. doi:10.1016/j.cell.2013.10.046

Caudron F, Barral Y. 2009. Septins and the lateral compartmentalization of eukaryotic membranes. Dev Cell 16:493-506. doi:10.1016/j.devcel.2009.04.003

Clay L, Caudron F, Denoth-Lippuner A, Boettcher B, Buvelot Frei S, Snapp EL, Barral Y. 2014. A sphingolipid-dependent diffusion barrier confines ER stress to the yeast mother cell. eLife 3:e01883. doi:10.7554/eLife.01883

Coustou V, Deleu C, Saupe S, Begueret J. 1997. The protein product of the het-s heterokaryon incompatibility gene of the fungus Podospora anserina behaves as a prion analog. Proc Natl Acad Sci 94:9773-9778. doi:10.1073/pnas.94.18.9773

Denoth-Lippuner A, Krzyzanowski MK, Stober C, Barral Y. 2014. Role of SAGA in the asymmetric segregation of DNA circles during yeast ageing. elife $\mathbf{3}$. doi:10.7554/eLife.03790

Derdowski A, Sindi SS, Klaips CL, DiSalvo S, Serio TR. 2010. A size threshold limits prion transmission and establishes phenotypic diversity. Science 330:680-683. doi:10.1126/science.1197785

Derkatch IL, Chernoff YO, Kushnirov VV, Inge-Vechtomov SG, Liebman SW. 1996. Genesis and variability of [PSI] prion factors in Saccharomyces cerevisiae. Genetics 144:13751386.

Ewers H, Tada T, Petersen JD, Racz B, Sheng M, Choquet D. 2014. A Septin-Dependent Diffusion Barrier at Dendritic Spine Necks. PloS One 9:e113916. doi:10.1371/journal.pone.0113916

Ferreira PC, Ness F, Edwards SR, Cox BS, Tuite MF. 2001. The elimination of the yeast [PSI+] prion by guanidine hydrochloride is the result of Hsp104 inactivation. Mol Microbiol 40:1357-1369.

Garì E, Volpe T, Wang H, Gallego C, Futcher B, Aldea M. 2001. Whi3 binds the mRNA of the G1 cyclin CLN3 to modulate cell fate in budding yeast. Genes Dev 15:2803-2808. doi:10.1101/gad.203501

Goedert M. 2015. Alzheimer's and Parkinson's diseases: The prion concept in relation to assembled $A \beta$, tau, and $\alpha$-synuclein. Science 349:1255555. doi:10.1126/science.1255555

Graziano BR, DuPage AG, Michelot A, Breitsprecher D, Moseley JB, Sagot I, Blanchoin L, Goode BL. 2011. Mechanism and cellular function of Bud6 as an actin nucleationpromoting factor. Mol Biol Cell 22:4016-4028. doi:10.1091/mbc.E11-05-0404 
706

707

708

709

710

711

712

713

714

715

716

717

718

719

720

721

722

Graziano BR, Jonasson EM, Pullen JG, Gould CJ, Goode BL. 2013. Ligand-induced activation of a formin-NPF pair leads to collaborative actin nucleation. J Cell Biol 201:595-611. doi:10.1083/jcb.201212059

Harvey ZH, Chen Y, Jarosz DF. 2018. Protein-Based Inheritance: Epigenetics beyond the Chromosome. Mol Cell 69:195-202. doi:10.1016/j.molcel.2017.10.030

Hou F, Sun L, Zheng H, Skaug B, Jiang Q-X, Chen ZJ. 2011. MAVS forms functional prion-like aggregates to activate and propagate antiviral innate immune response. Cell 146:448-461. doi:10.1016/j.cell.2011.06.041

Hu Q, Milenkovic L, Jin H, Scott MP, Nachury MV, Spiliotis ET, Nelson WJ. 2010. A septin diffusion barrier at the base of the primary cilium maintains ciliary membrane protein distribution. Sci N Y NY 329:436-439. doi:10.1126/science.1191054

Janke C, Magiera MM, Rathfelder N, Taxis C, Reber S, Maekawa H, Moreno-Borchart A, Doenges G, Schwob E, Schiebel E, Knop M. 2004. A versatile toolbox for PCR-based tagging of yeast genes: new fluorescent proteins, more markers and promoter substitution cassettes. Yeast Chichester Eng/ 21:947-962. doi:10.1002/yea.1142

Jarosz DF, Brown JCS, Walker GA, Datta MS, Ung WL, Lancaster AK, Rotem A, Chang A, Newby GA, Weitz DA, Bisson LF, Lindquist S. 2014. Cross-kingdom chemical communication drives a heritable, mutually beneficial prion-based transformation of metabolism. Cell 158:1083-1093. doi:10.1016/j.cell.2014.07.025

Khan MR, Li L, Pérez-Sánchez C, Saraf A, Florens L, Slaughter BD, Unruh JR, Si K. 2015. Amyloidogenic Oligomerization Transforms Drosophila Orb2 from a Translation Repressor to an Activator. Cell 163:1468-1483. doi:10.1016/j.cell.2015.11.020

Kim SK, Shindo A, Park TJ, Oh EC, Ghosh S, Gray RS, Lewis RA, Johnson CA, Attie-Bittach T, Katsanis N, Wallingford JB. 2010. Planar cell polarity acts through septins to control collective cell movement and ciliogenesis. Sci N Y NY 329:1337-1340. doi:10.1126/science.1191184

Lang GI, Murray AW. 2008. Estimating the per-base-pair mutation rate in the yeast Saccharomyces cerevisiae. Genetics 178:67-82. doi:10.1534/genetics.107.071506

Luedeke C, Frei SB, Sbalzarini I, Schwarz H, Spang A, Barral Y. 2005. Septin-dependent compartmentalization of the endoplasmic reticulum during yeast polarized growth. $J$ Cell Biol 169:897-908. doi:10.1083/jcb.200412143

Moore SA. 1984. Yeast cells recover from mating pheromone alpha factor-induced division arrest by desensitization in the absence of alpha factor destruction. $J$ Biol Chem 259:1004-1010.

Mostowy S, Cossart P. 2012. Septins: the fourth component of the cytoskeleton. Nat Rev Mol Cell Biol 13:183-194. doi:10.1038/nrm3284

Narayanan S, Walter S, Reif B. 2006. Yeast prion-protein, sup35, fibril formation proceeds by addition and substraction of oligomers. Chembiochem Eur J Chem Biol 7:757-765. doi:10.1002/cbic.200500382

Prusiner SB. 1982. Novel proteinaceous infectious particles cause scrapie. Sci $N$ Y NY 216:136-144.

Saarikangas J, Barral Y. 2011. The emerging functions of septins in metazoans. EMBO Rep 12:1118-1126. doi:10.1038/embor.2011.193

Saarikangas J, Caudron F, Prasad R, Moreno DF, Bolognesi A, Aldea M, Barral Y. 2017. Compartmentalization of ER-Bound Chaperone Confines Protein Deposit Formation to the Aging Yeast Cell. Curr Biol CB 27:773-783. doi:10.1016/j.cub.2017.01.069 
Serio TR, Cashikar AG, Kowal AS, Sawicki GJ, Moslehi JJ, Serpell L, Arnsdorf MF, Lindquist SL. 2000. Nucleated Conformational Conversion and the Replication of Conformational Information by a Prion Determinant. Science 289:1317-1321. doi:10.1126/science.289.5483.1317

Sharma SV, Agatsuma T, Nakano H. 1998. Targeting of the protein chaperone, HSP90, by the transformation suppressing agent, radicicol. Oncogene 16:2639-2645. doi:10.1038/sj.onc.1201790

Shcheprova Z, Baldi S, Frei SB, Gonnet G, Barral Y. 2008. A mechanism for asymmetric segregation of age during yeast budding. Nature 454:728-734. doi:10.1038/nature07212

Si K, Choi Y-B, White-Grindley E, Majumdar A, Kandel ER. 2010. Aplysia CPEB can form prionlike multimers in sensory neurons that contribute to long-term facilitation. Cell 140:421-435. doi:10.1016/j.cell.2010.01.008

Suzuki G, Shimazu N, Tanaka M. 2012. A Yeast Prion, Mod5, Promotes Acquired Drug Resistance and Cell Survival Under Environmental Stress. Science 336:355-359. doi:10.1126/science.1219491

Toure A, Rode B, Hunnicutt GR, Escalier D, Gacon G. 2011. Septins at the annulus of mammalian sperm. Biol Chem 392:799-803. doi:10.1515/BC.2011.074

True HL, Lindquist SL. 2000. A yeast prion provides a mechanism for genetic variation and phenotypic diversity. Nature 407:477-483. doi:10.1038/35035005

Tuite MF. 2016. Remembering the Past: A New Form of Protein-Based Inheritance. Cell 167:302-303. doi:10.1016/j.cell.2016.09.036

Tuite MF, Cox BS. 2006. The [PSI+] prion of yeast: a problem of inheritance. Methods San Diego Calif 39:9-22. doi:10.1016/j.ymeth.2006.04.001

Tuite MF, Mundy CR, Cox BS. 1981. Agents that cause a high frequency of genetic change from [psi+] to [psi-] in Saccharomyces cerevisiae. Genetics 98:691-711.

Vergés E, Garì E, Gallego C, Aldea M. 2007. Cyclin Cln3 is retained at the ER and released by the J chaperone Ydj1 in late G1 to trigger cell cycle entry. 26:649-662. doi:10.1016/j.molcel.2007.04.023

Wickner RB, Shewmaker FP, Bateman DA, Edskes HK, Gorkovskiy A, Dayani Y, Bezsonov EE. 2015. Yeast prions: structure, biology, and prion-handling systems. Microbiol Mol Biol Rev MMBR 79:1-17. doi:10.1128/MMBR.00041-14

Yuan AH, Hochschild A. 2017. A bacterial global regulator forms a prion. Sci N Y NY 355:198201. doi:10.1126/science.aai7776

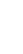

(


Figure Legends

767

768

769

770

771

772

773

774

775

776

777

778

779

780

781

782

783

784

785

786

787

788

789

790

791

792

793

794

795

796

797

798

799

Figure 1. A novel epigenetic phenotype, constitutive escapers. (A) Serial 1/10 dilutions of exponentially growing cultures of indicated strains spotted on a YPD solid medium (left) or YPD containing $\alpha$-factor (10nM middle and $600 \mathrm{nM}$ right). The bottom panel shows three bud6 6 independent clones with high frequency of CE. (B) Frequency of appearance of CEs in the indicated genotypes (median with 95\% confidence interval, $n>37$ clones for each, Dunn's multiple comparisons test was used to determine significance). (C) Distribution of the logit transformed ratios $(R)$ for each genotype. (D) Cell size distributions of individual clones or CE isolates of indicated strains ( $n>39$ clones for each). (E) Maximal projection images of sur2 $\Delta$ cells expressing Whi3-GFP from $20 \mathrm{CE}$ isolates and the parental strain. Isolates are in order of mean cell size from the smallest (orange frame are smaller than the blue framed parental strain) to the largest (purple are larger than the parental strain). Scale bar $=5 \mu \mathrm{m}$.

Figure 2. Endoplasmic reticulum compartmentalization by a lateral membrane diffusion barrier is not required for prion induction and curing. (A) Fluorescence intensity measured by flow cytometer of [PSI+] WT and [PSI+] sur2 $\Delta$ cells treated or not with $0.1 \mathrm{mM}$ and $1 \mathrm{mM}$ GuHCl. (B) Frequency of de novo [PSI+] appearance and (C) percentage of cells cured of [PSI+] over time. Graphs A and B display mean \pm SD.

Figure 3. Sup35 and Whi3 prion foci are not closely linked to ER membranes while Whi3 super-assemblies and granules are. (A) Single focal plane images of $[P S /+]$ cells expressing Sup35-GFP and Sec61-mCherry. (B) Percentage of Sup35 foci close to ER membranes (1180 foci analysed from 90 cells of three independent clones, mean $\pm S D$ is presented). (C) Percentage of cells with at least one Sup35-GFP focus away from ER membranes (3 clones with more than 90 cells each). (D) Single focal plane images of cells expressing Whi3-mNG and Sec61-mCherry exposed to pheromone for 3 hours (top left and middle left) and 4 hours (bottom left), and cells exposed for 5 hours to pheromone and released in a pheromone free medium then imaged at 6 hours (top right) and 10.5 hours (bottom right) after initial exposure to pheromone. (E) Percentage of Whi3-mNG super-assemblies close to ER membranes (3 independent clones, $>300$ super-assemblies from $>153$ cells were analysed, mean \pm SD are presented). (F) Percentage of cells with at least one Whi3-mNG super-assembly away from ER membranes (3 independent clones, $>300$ super-assemblies from >153 cells were analysed, mean $\pm S D$ are presented). (G) Single focal plane images of bud6 $\Delta$ cells (top) and bud6 $\triangle$ CE cells (bottom) expressing Whi3-mNG and Sec61-mCherry. (H) Percentage of Whi3mNG granules (bud6 6 ) and foci (bud6 6 CE) localizing close to ER membranes (4 clones or isolates, 527 granules and 359 foci in 200 cells were analysed, mean $\pm S D$ are presented). For 
800

801

802

803

804

805

806

807

808

809

810

811

812

813

814

815

816

817

818

819

820

821

822

823

824

825

826

827

828

829

830

831

all panel, arrows point at foci away from the ER and arrowheads point at foci close to the ER. Scale bars $=5 \mu \mathrm{m}$.

Figure 4. Whi3-3GFP super-assemblies form in the buds of mutants with a weak diffusion barrier. (A) Single focal plane images of Whi3-3GFP expressing cells. Scale bar = $5 \mu \mathrm{m}$. (B) Quantification of buds with a detectable Whi3-3GFP super-assembly $(n=122$ cells,

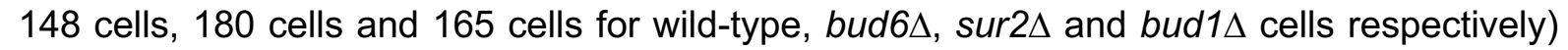
Results from bud6 6 , sur $2 \Delta$ and bud $1 \Delta$ are significantly different from wild type $(p<0.0001$, oneway ANOVA).

Figure 5. The ER diffusion barrier prevents daughter cells from inheriting the pheromone refractory state. (A) Escape of a wild-type, a bud6 $\Delta$, a sur2 $\Delta$ and a bud $1 \Delta$ cell exposed to $7 \mathrm{nM}$ pheromone. (B) Percentage of initial cells still shmooing after the indicated time ( $n>154$ cells). (C) Percentage of daughter cells budding immediately after separation from the mother cell $(n>128$ cells, $n>121$ cells, $n>111$ cells, $n>88$ cells and $n>59$ cells for the $1^{\text {st }}, 2^{\text {nd }}, 3^{\text {rd }}, 4^{\text {th }}$ and $5^{\text {th }}$ daughter respectively). (D) Timing of escape of daughter cells that shmooed (sur2 $\Delta$ is significantly different from wild type, $p<0.0001$, one-way ANOVA, all other comparisons are not significantly different, n=366 cells, 81 cells, 416 cells and 193 cells for wild-type, bud6 $\Delta$, sur2 $\Delta$ and bud1 $1 \Delta$ cells respectively).

Figure 6. The polyQ domain of Whi3 is required for inheritance of the pheromone refractory state by diffusion barrier impaired daughter cells. Percentage of daughter cells of indicated genotypes budding immediately after separation from the mother cell. Mean $\pm S D$ are presented.

\section{Supplemental Figure 1. Deviation of the residuals from a cumulative normal distribution.}

Supplemental Figure 2. Non-mendelian inheritance of the CE phenotype during meiosis. Schematic of the experimental test (left) and results (right) of 2 backcrosses of sur2 $\Delta$ parental strain (pooled in one line) and 13 CE backcrossed.

Supplemental Figure 3. The CE phenotype can be partially cured by Ssa1 inhibition. Schematic of the experimental test. CE were isolated, restreaked 3 times on YPD, YPD + radicicol $(10 \mu \mathrm{M})$ or YPD $+\mathrm{GuHCl}(3 \mathrm{mM})$ or transformed with a plasmid expressing SSA $1^{D N}$ and restreaked 3 times on selective medium. CE were then tested again on YPD + alpha factor $(0.6 \mu \mathrm{M})$. The right panels display microscopic images of the parental sur2 $\Delta$ strain and 
bioRxiv preprint doi: https://doi.org/10.1101/2020.03.13.990119; this version posted March 20, 2020. The copyright holder for this preprint (which was not certified by peer review) is the author/funder, who has granted bioRxiv a license to display the preprint in perpetuity. It is made available under aCC-BY-NC-ND 4.0 International license.

832 CE1 and CE2 on YPD + alpha factor $(0.6 \mu \mathrm{M})$ after the corresponding treatments. Arrows point 833 at shmooing cells in CE1 transformed with a plasmid expressing $S S A 1^{D N}$. Scale bar $=10 \mu \mathrm{m}$. 
Figure 2
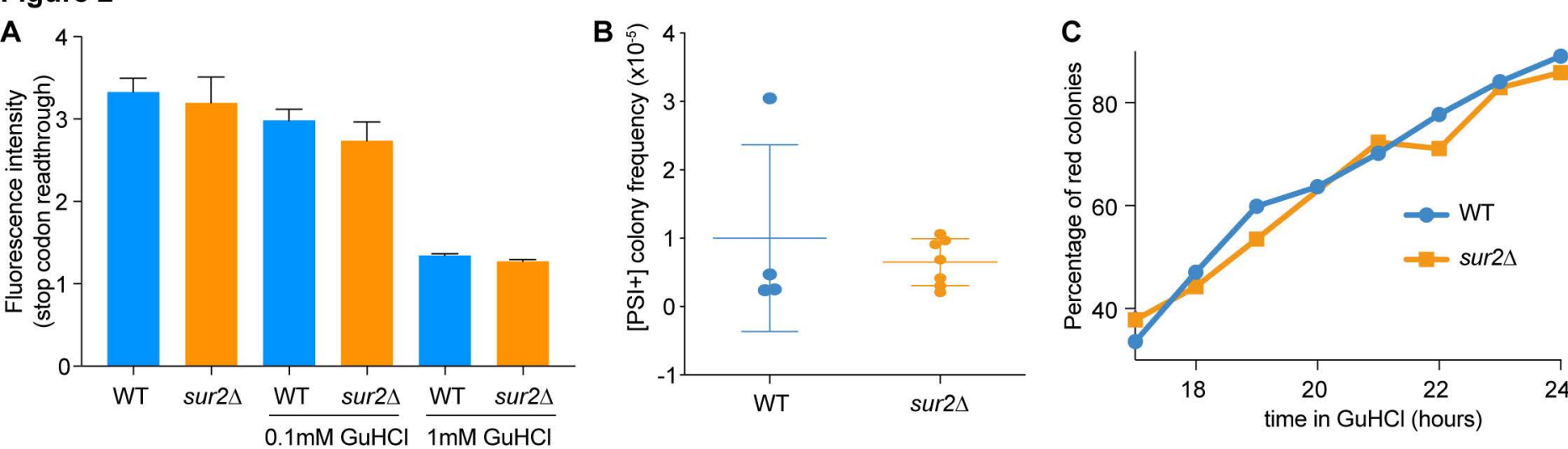
Figure 3

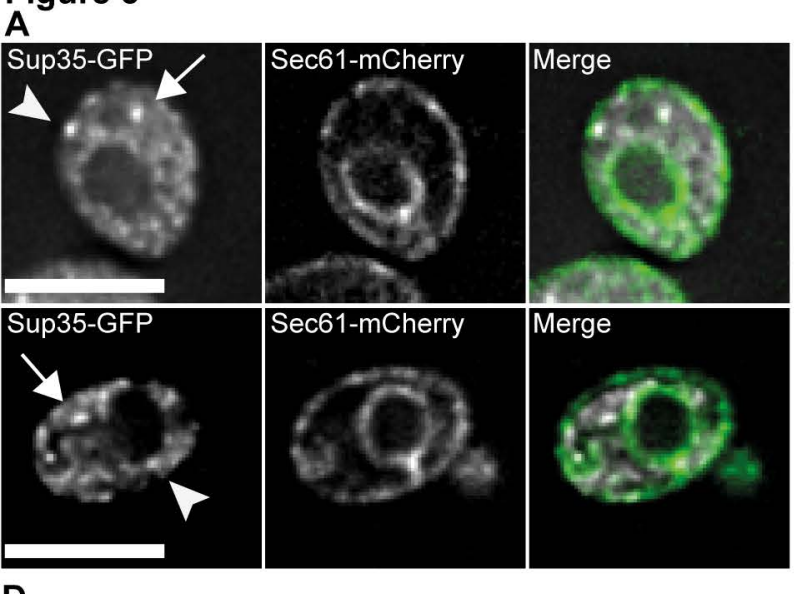

\section{D}

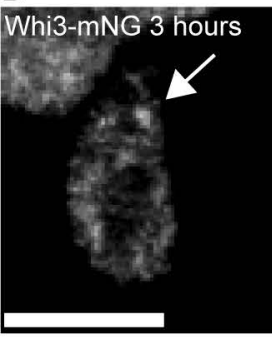

Whi3-mNG 3 hours

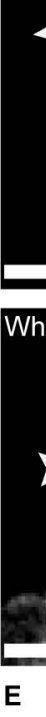

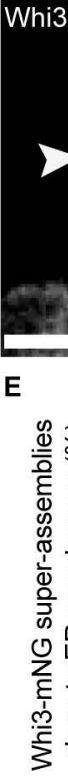

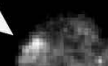

5)

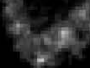

mNG 4 hours

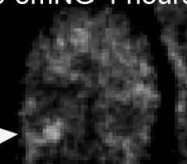

Ext

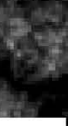

45

$\sqrt{3}$

$+3$

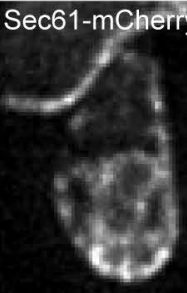

Sec61-mCherry

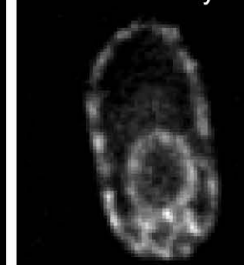

Merge
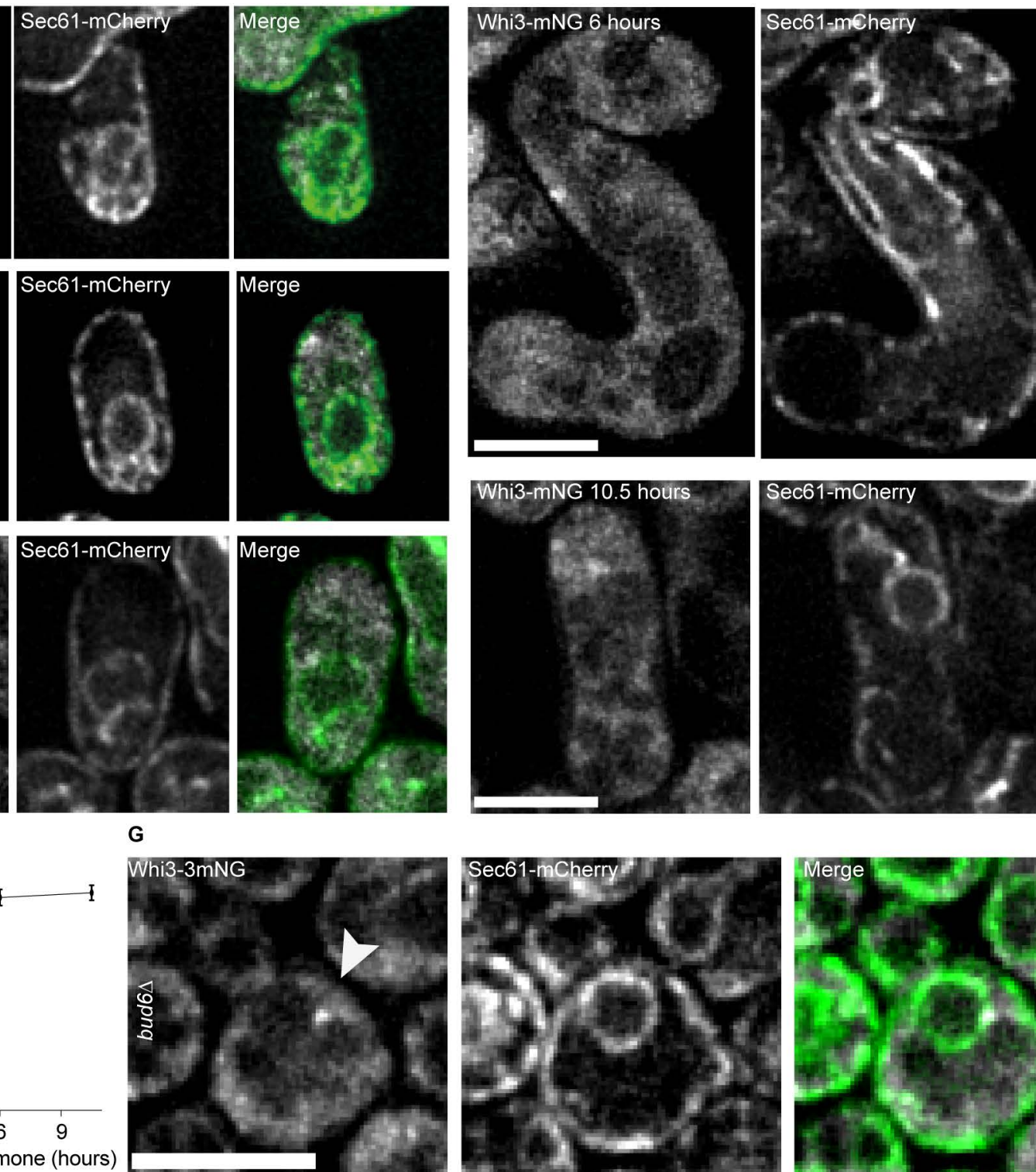

Time in pheromone (hours)
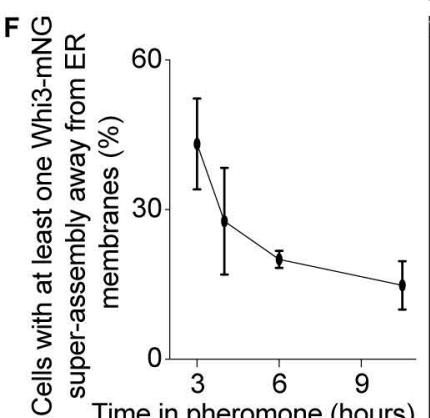
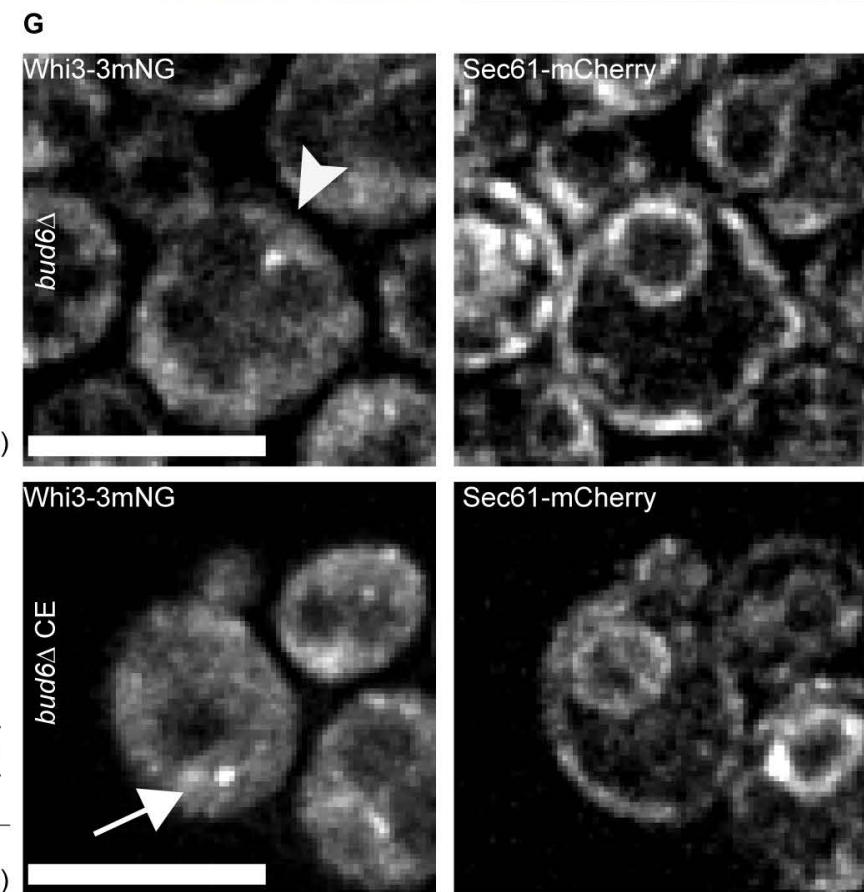

Sec61-mCherry

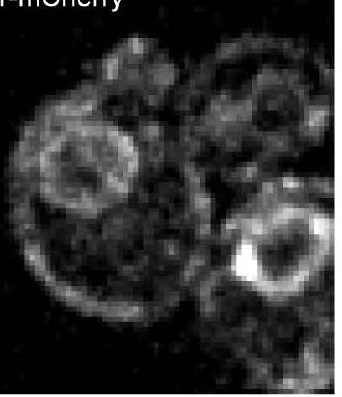

C

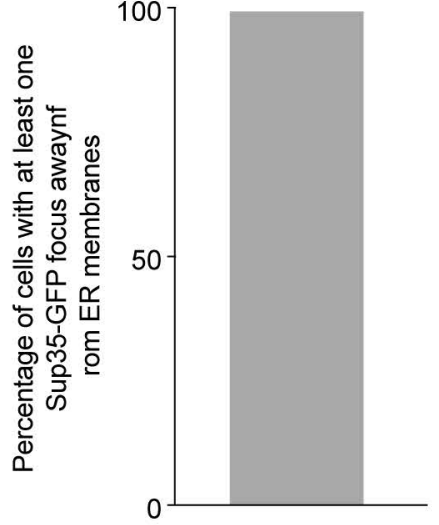

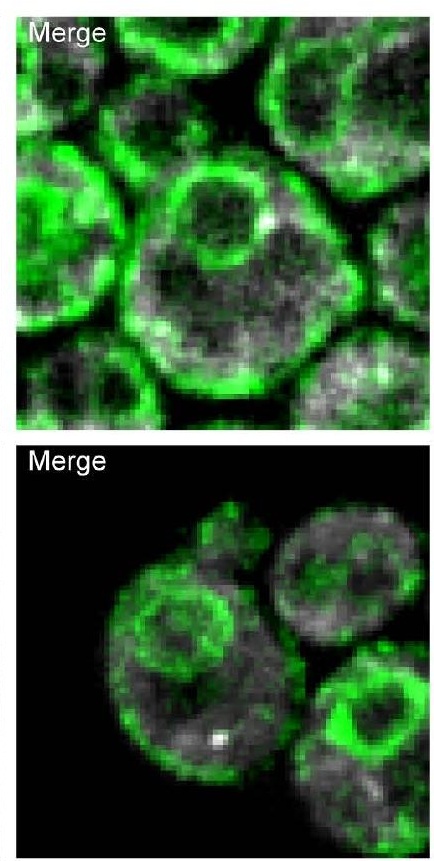

H

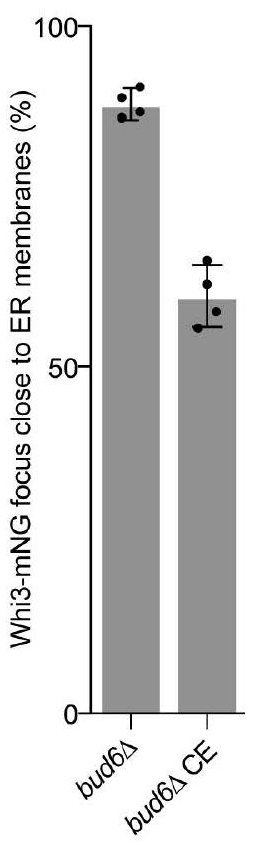


Figure 4
A Wild Tvpe

bud6s

sur2A

bud1s

B

(10)

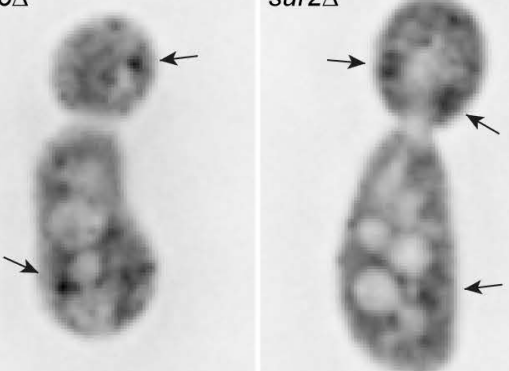

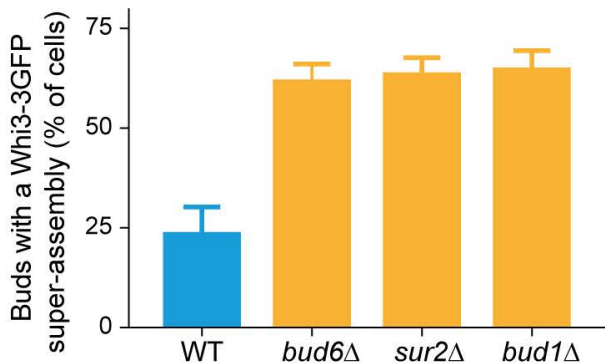


Normal Q-Q

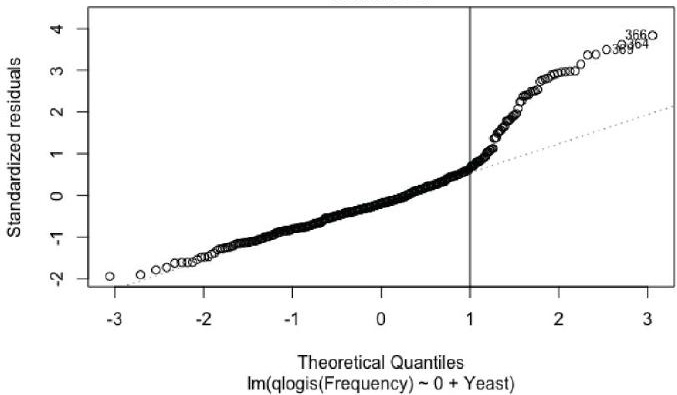


CE backcrossed

Meiosis

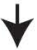

Tetrad dissection

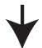

tetrads

\section{$\begin{array}{llll}1 & 2 & 3 & 4\end{array}$}

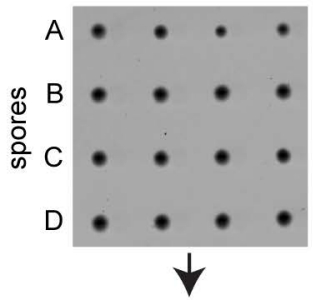

Restreak on YPD

+ alpha-factor $(0.6 \mu \mathrm{M})$

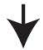

Growth pattern

2

3

4

Number of tetrads with growth pattern on YPD + alpha-factor $(0.6 \mu \mathrm{M})$

\begin{tabular}{|c|c|c|c|}
\hline sur2 & 10 & 0 & 0 \\
\hline$C E 1$ & 4 & 0 & 0 \\
\hline CE2 & 3 & 1 & 0 \\
\hline CE3 & 3 & 1 & 0 \\
\hline CE4 & 4 & 0 & 0 \\
\hline CE5 & 19 & 3 & 2 \\
\hline CE6 & 17 & 8 & 0 \\
\hline CE7 & 9 & 3 & 1 \\
\hline CE8 & 2 & 8 & 2 \\
\hline$C E 9$ & 4 & 0 & 0 \\
\hline$C E 10$ & 7 & 4 & 1 \\
\hline CE11 & 11 & 1 & 0 \\
\hline CE12 & 11 & 0 & 0 \\
\hline CE13 & 33 & 0 & 0 \\
\hline
\end{tabular}


YPD + alpha Factor $(0.6 \mu M)$

sur2 $\Delta$

$\begin{array}{ll}\text { CE1 } & \text { CE2 }\end{array}$

\begin{tabular}{|l|l|l|l|}
\hline Sur2a & $C E 1$ & $C E 2$ & $C E 3$ \\
\hline
\end{tabular} \begin{tabular}{|l|llllll}
\hline CE4 & CE5 & CE6 & CE7 & CE8 & CE9 \\
\hline
\end{tabular} \begin{tabular}{llllll}
\hline$C E 10$ & CE11 CE12 & CE13 & CE14 & CE15 \\
\hline
\end{tabular} CE16 CE17 CE 18 CE 19 CE20 CE21 \begin{tabular}{llllll}
\hline CE22 & CE233 & CE24 & CE25 & CE26 & CE27 \\
\hline
\end{tabular} CE28 CE29 CE30 CE31

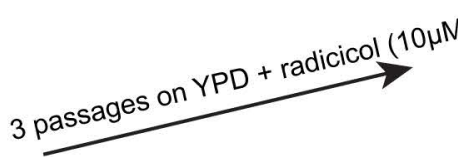

(10 $\mu \mathrm{M})$
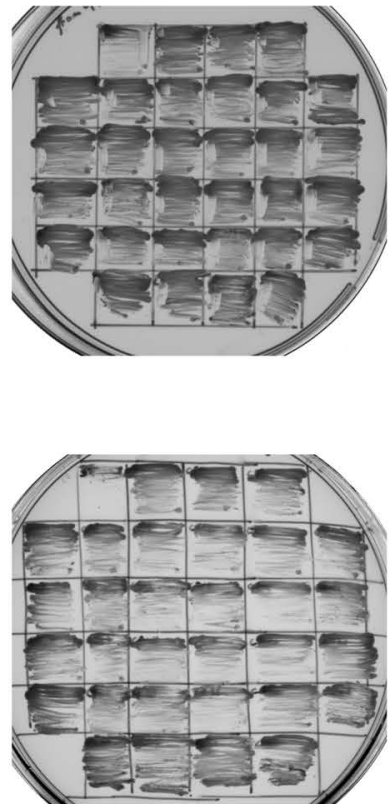
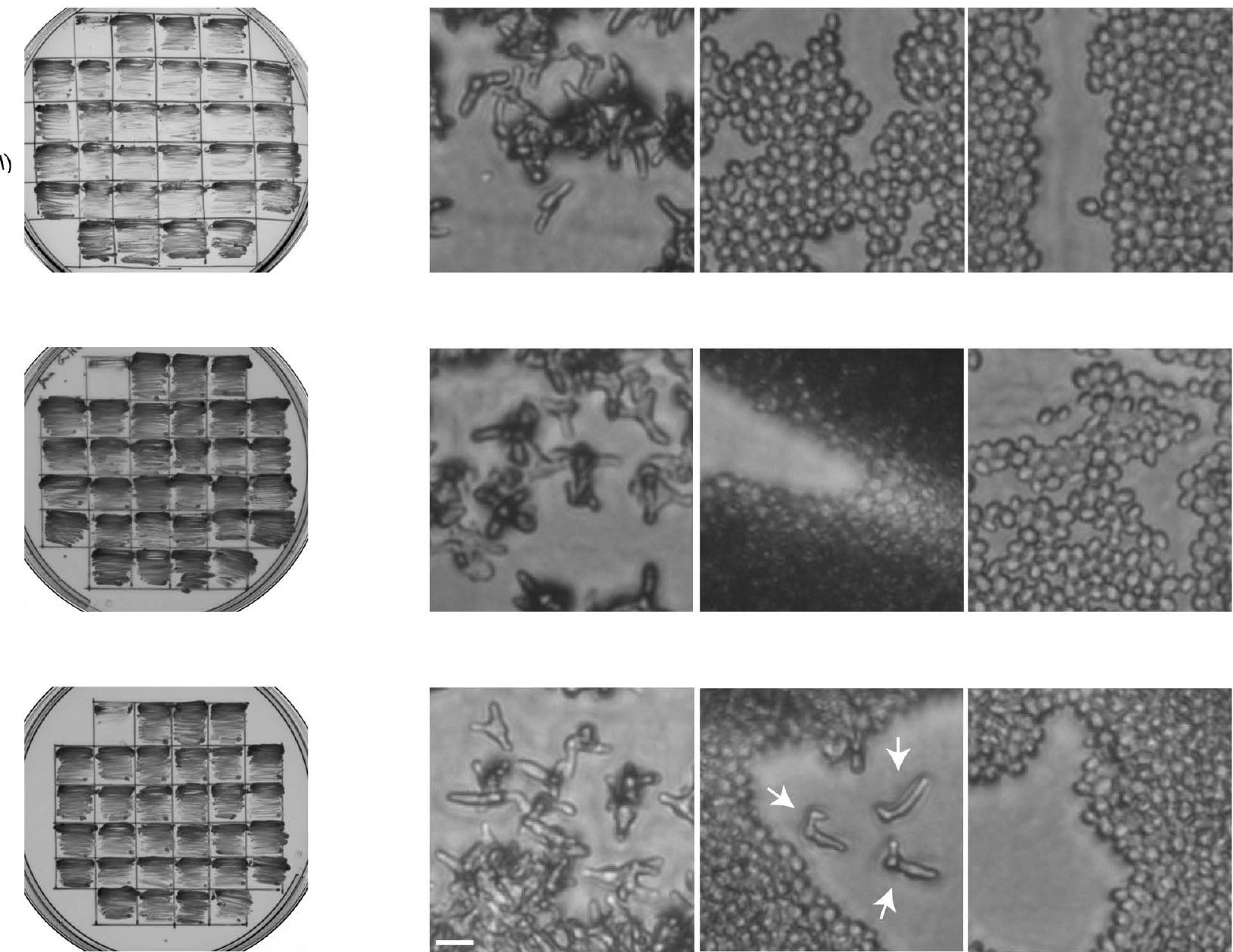

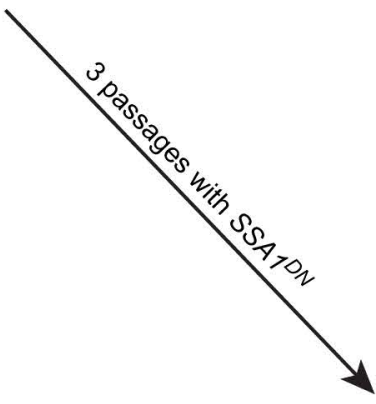

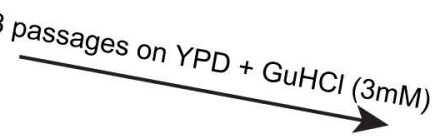
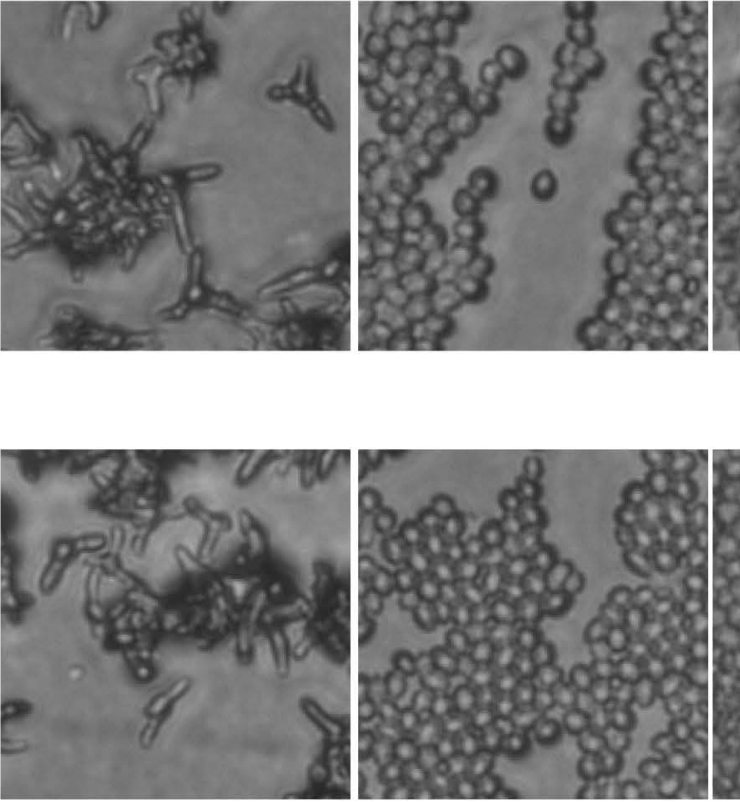

$0<8$

25, ts 3858 \& coso $82+39$ 